\title{
Downstaging of Hepatocellular Carcinoma Prior to Liver Transplant: Is There a Role for Adjuvant Sorafenib in Locoregional Therapy?
}

\author{
Parsia A. Vagefi • Ryutaro Hirose
}

Published online: 5 May 2010

(C) The Author(s) 2010. This article is published with open access at Springerlink.com

\begin{abstract}
Hepatocellular carcinoma (HCC) remains a common cause of mortality worldwide. Liver transplantation has emerged as the optimal treatment for cirrhotic patients with HCC; however, the shortage of donor organs leaves waitlisted patients at risk for disease progression beyond transplant criteria. Prevention of waitlist dropout has fueled investigation into a wide array of locoregional therapies for the management of HCC in candidates awaiting liver transplantation. We present a patient with HCC who underwent treatment with sorafenib, which resulted in a remarkable reduction in tumor burden to allow for liver transplant listing.
\end{abstract}

Keywords hepatocellular cancer - liver transplantation . locoregional therapy $\cdot$ sorafenib

\section{Case Report}

A 54-year-old gentleman with cirrhosis and end-stage liver disease secondary to chronic hepatitis $\mathrm{C}$ presented for evaluation for liver transplantation. The patient was first diagnosed with hepatitis C in 1990, and subsequently diagnosed with cirrhosis in 1991 by liver biopsy. At that time he was briefly treated with interferon, but sustained a severe allergic reaction following his fourth dose prompting

\footnotetext{
P. A. Vagefi $\cdot$ R. Hirose

Division of Transplantation, University of California,

San Francisco, CA, USA

P. A. Vagefi $(\bowtie)$

Department of Surgery, University of California, San Francisco,

505 Parnassus Ave,

San Francisco, CA 94143, USA

e-mail: parsia.vagefi@ucsfmedctr.org
}

discontinuation. In February of 2007, he developed worsening fatigue and sought care from his primary care physician. A computed tomographic (CT) scan was performed which demonstrated a small, irregular-appearing liver consistent with cirrhosis, but no liver masses were appreciated and the portal venous system was patent (Fig. 1a). An alphafetoprotein (AFP) level was noted to be elevated at $7,100 \mathrm{ng} / \mathrm{ml}$ in September of 2007. A CT scan was repeated in October of 2007 and demonstrated thrombus within the left portal vein (Fig. 1b). Magnetic resonance imaging of the liver was performed and demonstrated cirrhosis with left portal vein occlusion. A heterogeneous nodular mass had replaced the entire left lobe of the liver and the caudate lobe of the liver with evidence of increased enhancement. There was another heterogeneous lesion with abnormally increased signal intensity in the medial segment of the left lobe measuring $2 \mathrm{~cm}$ in diameter. Both of these lesions were highly suspicious for hepatocellular carcinoma (HCC). A metastatic evaluation was negative and a repeat AFP level was $24,100 \mathrm{ng} / \mathrm{ml}$. The patient was referred to an outside institution for transplant evaluation, and was deemed unsuitable due to evidence of an infiltrating form of HCC with evidence of portal vein thrombosis. By December of 2007, the patient's AFP level had increased to $194,000 \mathrm{ng} / \mathrm{ml}$. A CT scan at that time demonstrated extensive thrombus within the main portal vein near the bifurcation that extended into the right and left portal veins. Sorafenib was started at a dose of $400 \mathrm{mg}$ twice daily in January of 2008. The AFP level decreased to $84,800 \mathrm{ng} / \mathrm{ml}$ and continued to exhibit a remarkable decline to $7,300 \mathrm{ng} / \mathrm{ml}$ by February of 2008, and $117 \mathrm{ng} / \mathrm{ml}$ by March of 2008 . As the patient developed leukopenia and thrombocytopenia, his sorafenib dose was reduced to $200 \mathrm{mg}$ twice daily. By April of 2008 this was further reduced to $200 \mathrm{mg}$ a day, and ultimately to $200 \mathrm{mg}$ twice weekly, on which he is currently maintained. By May 
Fig. $1 \mathrm{CT}$ scan of the abdomen July 2007 demonstrating a cirrhotic liver, and no evidence of portal thrombus (a). Followup CT scan of the abdomen performed October 2007 demonstrating thrombus within the left portal vein (b, arrow)
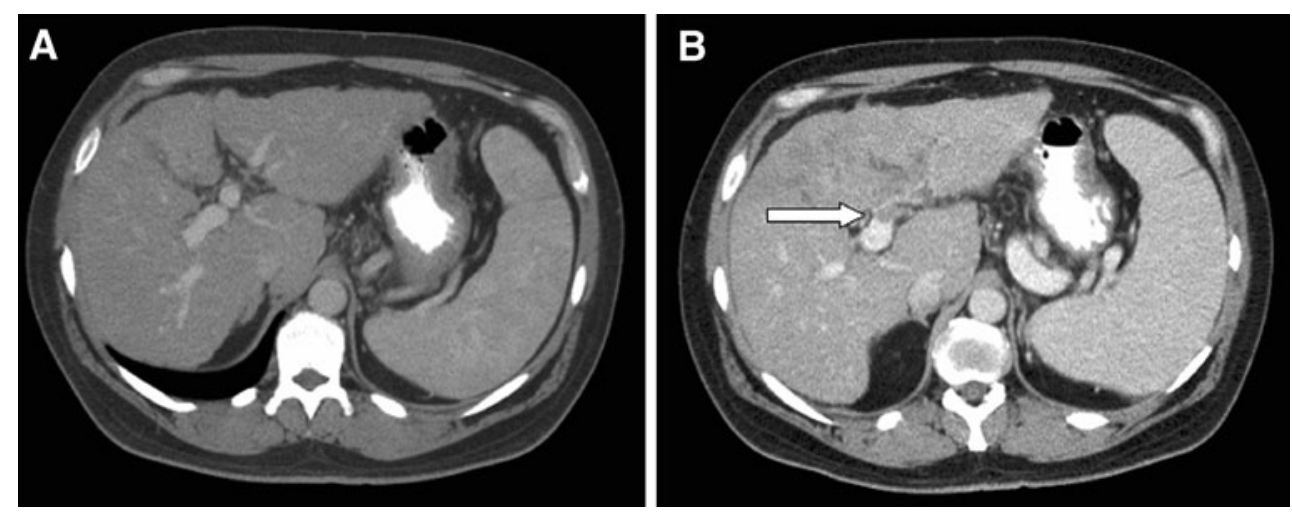

of 2008, his AFP level was $3.7 \mathrm{ng} / \mathrm{ml}$, and subsequent testing at monthly intervals have shown a persistently low AFP level between 3 and $5 \mathrm{ng} / \mathrm{ml}$ (Table 1).

The patient was subsequently referred to our institution for consideration for transplantation in March of 2009. On evaluation he reported mental slowing and difficulty with memory, and his physical examination was significant for ascites and peripheral edema. Laboratory testing at that time was significant for a platelet count of 57,000, an International Normalized Ratio of 1.4, serum albumin of 3.0, total bilirubin of 2.1, AST of 76, ALT of 68, alkaline phosphatase of 50, and an AFP level of $3.4 \mathrm{ng} / \mathrm{ml}$. His calculated Model for End-Stage Liver Disease (MELD) Score was 13 . The patient underwent exploratory laparoscopy and was found to have a grossly cirrhotic liver with no definitive mass appreciated. Intraoperative laparoscopic ultrasound examination of the liver did visualize an area in segment 4 that consisted of a nest of abnormal blood vessels. This area was thoroughly biopsied, following which radiofrequency ablation was performed due to concern for malignancy. Notably, all of the liver biopsies demonstrated cirrhosis with focal scar and chronic inflammation, and no evidence of carcinoma. Follow-up imaging in October of 2009 demonstrated a well-defined area of peripheral low density involving

Table 1 AFP levels in a patient with HCC

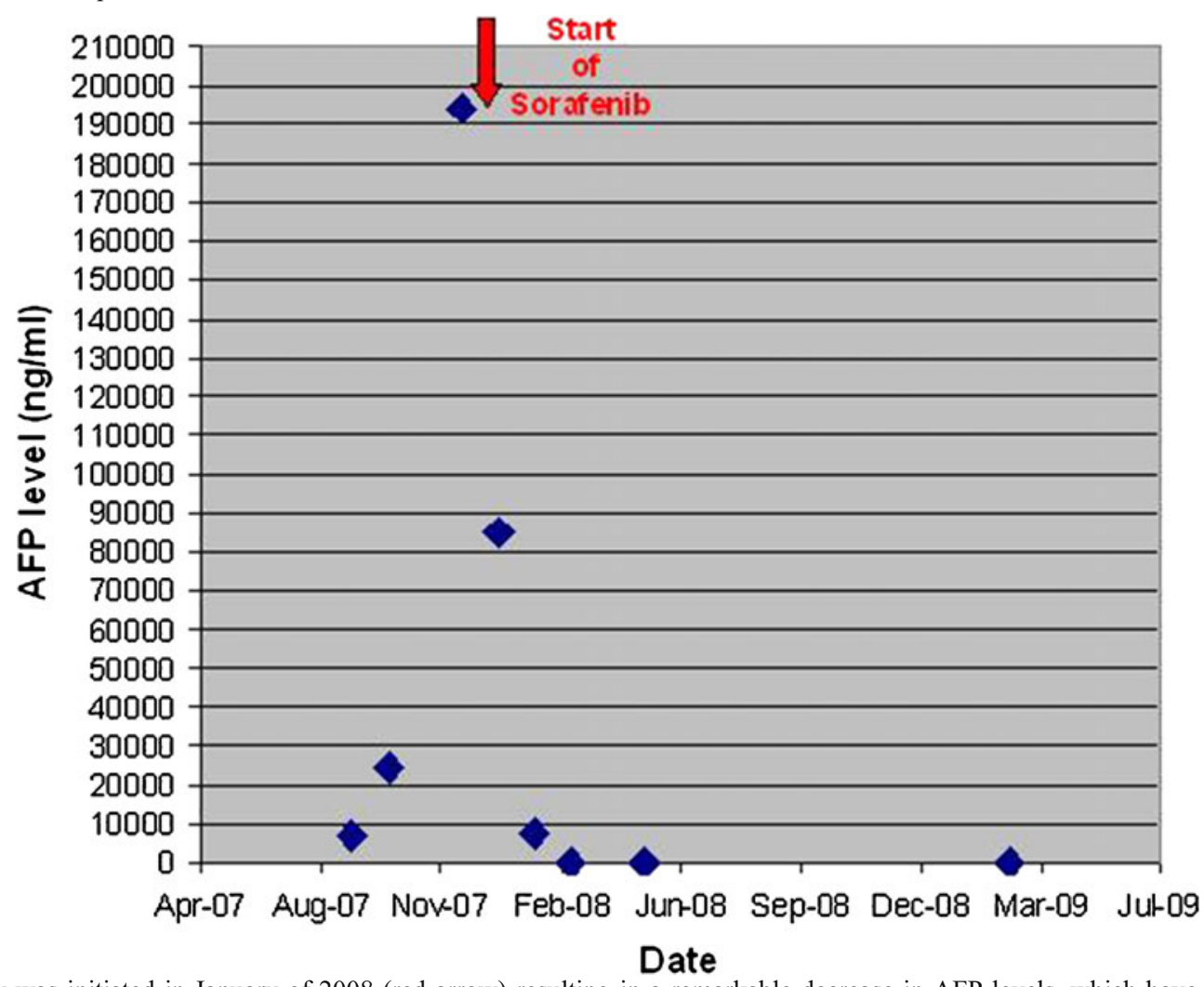

Sorafenib therapy was initiated in January of 2008 (red arrow) resulting in a remarkable decrease in AFP levels, which have persisted 
the anterior aspect of segment 4 of the liver, which corresponded to the site of prior RFA ablation. Just medial to this ablation site was an area of more ill-defined low density, which was suspicious for residual/recurrent tumor. A non-occlusive thrombus involving the splenic vein, extending to the portosplenic confluence was noted. In addition, nonopacification of the left portal vein, with numerous associated collaterals consistent with cavernous transformation, was appreciated. The main portal vein and right portal vein were patent. As the patient's tumor burden decreased to within the Milan criteria, and as he had demonstrated stability at that tumor burden, the patient was subsequently listed for transplantation with MELD exception points.

\section{Discussion}

Hepatocellular carcinoma (HCC) is the third most common cause of cancer mortality worldwide, with a 626,000 new cases reported yearly [1]. The incidence of HCC remains high in Asia and parts of Africa, but there is clear evidence of increasing incidence in the USA and Europe, likely related, at least in part, to an increasing incidence of Hepatitis C infection. Although HCC can present in a patient with a noncirrhotic liver, cirrhosis remains the main risk factor that predisposes to the development of HCC.

Although surgical resection remains an accepted form of therapy for noncirrhotic patients with HCC, liver transplantation currently remains the optimal treatment for the cirrhotic patient. Early results with liver transplantation were unfavorable, plagued by poor survival rates and a high incidence of post-transplantation recurrence [2]. It was not until 1996, when Mazzaferro et al. defined a subset of patients with unresectable HCC for whom liver transplantation was the appropriate treatment, now referred to as the "Milan criteria" [3]. Transplantation within the Milan criteria (single tumor $\leq 5 \mathrm{~cm}$ in diameter, or two to three tumors $\leq 3 \mathrm{~cm}$ in diameter) resulted in overall and recurrence-free survival rates of $85 \%$ and $92 \%$, respectively, at 4 years.

Liver transplantation for $\mathrm{HCC}$ with tumor burden within the Milan criteria has gained wide acceptance as a legitimate, and optimal, treatment. The excellent results obtained subsequently prompted further investigation into whether the Milan criteria could be expanded to include patients with a larger HCC tumor burden. In 2001, Yao et al. proposed expansion of the Milan criteria for HCC liver transplant candidates [4]. Termed the UCSF criteria, these patients had a single tumor $\leq 6.5 \mathrm{~cm}$ in diameter, or two or three tumors none exceeding $4.5 \mathrm{~cm}$ in diameter and whose sum of tumor diameters did not exceed $8 \mathrm{~cm}$. Patient transplanted within the UCSF criteria demonstrated 1- and 5 -year survival rates of $90 \%$ and $75 \%$, respectively, results which were equivalent when compared with survival rates of those transplanted within the Milan criteria.

The shortage of organs, in conjunction with the risk of HCC progression beyond the Milan criteria prompting dropout from the liver transplant waitlist, has fueled investigation into a wide array of locoregional therapies for the management of HCC in candidates awaiting liver transplantation. These therapies range from surgical resection, cryotherapy, radiofrequency ablation, percutaneous ethanol injection, microwave ablation, interstitial laser coagulation, and transarterial chemoembolization. Locoregional therapy has been proposed as a strategy to not only retard HCC progression and prevent dropout from the transplant waitlist, but also as a means of downstaging patients to within Milan criteria, and thus achieve eligibility for transplantation [5]. In addition, pre-transplant locoregional therapy may serve a role in improving survival following liver transplantation for HCC [6]. Data from the Scientific Registry of Transplant Recipients demonstrated improved patient and graft survival rates at 3 years for patients with HCC treated with pre-operative locoregional therapy, when compared to untreated patients (patient survival, $79 \%$ versus $75 \%, p=0.03$; graft survival, $76 \%$ versus $71 \%, p=0.03$ ) [7]. The optimal form of locoregional therapy has yet to be determined. Indeed, to achieve complete tumor necrosis may require multiple sessions, or even multiple forms, of locoregional therapy. Therapy not only serves as a bridge for those patients within the Milan criteria awaiting transplantation, but also may allow for those patients with tumors beyond the Milan criteria to obtain eligibility for transplantation by demonstrating a response to locoregional therapy.

HCC is highly refractory to traditional cytotoxic chemotherapy, with no evidence to date of a survival benefit from its use $[8,9]$. The relatively chemo-resistant phenotype has prompted further investigation into the use of novel small molecule drugs for improved clinical efficacy in the treatment of HCC. Recently, the SHARP trial, a large randomized phase III study of patients with biopsy-proven advanced HCC, was completed. In this study, patients were randomized to receive either oral sorafenib or a placebo. Sorafenib (Nexavar, Bayer HealthCare PharmaceuticalsOnyx Pharmaceuticals), a small molecule multi-kinase inhibitor, acts via inhibition of tumor-cell proliferation and tumor angiogenesis, as well as augmentation of the rate of apoptosis. The SHARP trial was the first randomized systemic therapy trial in patients with advanced HCC which demonstrated an overall, albeit modest, survival benefit. Patients receiving sorafenib, versus the placebo group, demonstrated improvement in both overall survival (median 10.7 months versus 7.9 months) as well as time to progression (median 5.5 months versus 2.8 months) [10]. A second phase III study of sorafenib versus placebo was 
completed in an Asian-Pacific population, and also demonstrated significant improvement in overall survival (median 6.5 months versus 4.2 months) as well as progression-free survival (median 2.8 months versus 1.4 months) in patients treated with sorafenib versus placebo, respectively [11].

We present a remarkable case of HCC tumor response to adjuvant sorafenib therapy, with effective downstaging to allow for liver transplant listing. Although there has been demonstration of improved survival in patients with unresectable HCC treated with sorafenib, the tumor response rate of patients with HCC treated with sorafenib has remained low (2\%) [10]. However, it remains to be determined whether sorafenib, in conjunction with locoregional therapy, can prove to be either effective in preventing progression beyond the Milan criteria or facilitating downstaging to within the Milan criteria, in patients with HCC awaiting liver transplantation [12, 13].

Open Access This article is distributed under the terms of the Creative Commons Attribution Noncommercial License which permits any noncommercial use, distribution, and reproduction in any medium, provided the original author(s) and source are credited.

\section{References}

1. Parkin DM, Bray F, Ferlay J, Pisani P. Global cancer statistics, 2002. CA Cancer J Clin. 2005;55:74-108.

2. Roayaie K, Feng S. Allocation policy for hepatocellular carcinoma in the MELD era: room for improvement? Liver Transpl. 2007;13(11 Suppl 2):S36-43.

3. Mazzaferro V, Regalia E, Doci R, et al. Liver transplantation for the treatment of small hepatocellular carcinomas in patients with cirrhosis. N Engl J Med. 1996;334(11):693-9.
4. Yao FY, Ferrell L, Bass NM, Watson JJ, Bacchetti P, Venook A, et al. Liver transplantation for hepatocellular carcinoma: expansion of the tumor size limits does not adversely impact survival. Hepatology. 2001;6:1394-403.

5. Yao FY, Hirose R, LaBerge JM, et al. A prospective study on downstaging of hepatocellular carcinoma prior to liver transplantation. Liver Transpl. 2005;11:1505-14.

6. Bharat A, Brown DB, Crippin JS, et al. Pre-liver transplantation locoregional adjuvant therapy for hepatocellular carcinoma as a strategy to improve longterm survival. J Am Coll Surg. 2006;203 (4):411-20.

7. Freeman Jr RB, Steffick DE, Guidinger MK, Farmer DG, Berg CL, Merion RM. Liver and intestine transplantation in the United States, 1997-2006. Am J Transplant. 2008;8(pt 2):958-76.

8. Thomas Yau, Pierre Chan, Richard Epstein, Ronnie T. Poon Evolution of systemic therapy of advanced hepatocellular carcinoma. World J Gastroenterol. 2008;14(42):6437-41.

9. Lopez PM, Villanueva A, Llovet JM. Systematic review: evidence-based management of hepatocellular carcinoma-an updated analysis of randomized controlled trials. Aliment Pharmacol Ther. 2006;23:1535-47.

10. Llovet JM, Ricci S, Mazzaferro V, Hilgard P, Gane E, Blanc JF, et al. Sorafenib in advanced hepatocellular carcinoma. N Engl J Med. 2008;359:378-90.

11. Cheng AL, Kang YK, Chen Z, Tsao CJ, Qin S, Kim JS, et al. Efficacy and safety of sorafenib in patients in the Asia-Pacific region with advanced hepatocellular carcinoma: a phase III randomised, double-blind, placebo-controlled trial. Lancet Oncol. 2009;10(1):25-34.

12. Hoffmann K, Glimm H, Radeleff B, Richter G, Heining C, Schenkel I, et al. Prospective, randomized, double-blind, multicenter, Phase III clinical study on transarterial chemoembolization (TACE) combined with Sorafenib versus TACE plus placebo in patients with hepatocellular cancer before liver transplantationHeiLivCa [ISRCTN24081794]. BMC Cancer. 2008;8:349.

13. Vitale A, Volk ML, Pastorelli D, Lonardi S, Farinati F, Burra P, et al. Use of sorafenib in patients with hepatocellular carcinoma before liver transplantation: a cost-benefit analysis while awaiting data on sorafenib safety. Hepatology. 2010;51(1):165-73. 